American Journal of Applied Sciences 4 (2): 56-59, 2007

ISSN 1546-9239

(C) 2006 Science Publications

\title{
Design and Implementation of an Adaptive Control for a Robot
}

\author{
Iraj Hassanzadeh, Abbas Harifi and Farid Arvani \\ Center of Excellence for Mechatronics, University of Tabriz, Tabriz, Iran
}

\begin{abstract}
This research investigates the performance of an adaptive controller for five-bar linkage robot. The proposed controller was entirely independent on the physical specifications of the robot. The controller was examined as the dynamics of the mechanical system varies for various environment conditions, which make it an ambiguous system. In this study, the introduced controller was designed based on the governing ideal Euler-Lagrange equations on the robot but assessed using the on-line dynamic simulation of the mechanism for different target configurations which guarantees the high performance and effectiveness of the designed controller.
\end{abstract}

Key words: Adaptive control, five-bar linkage, robot, self tuning controller

\section{INTRODUCTION}

Self tuning controller should be very convenient way for nonlinear system such as robot manipulator. There are many references in this regard ${ }^{[1-10]}$. In our study, we proposed an adaptive controller which entirely independent of physical aspects of Manipulator such as center of mass, size and more importantly load variations. Also, the effectiveness of proposed method is investigated to overcome the sever nonlinearity of Robot dynamic problems. Although the results are not extensive, yet they can be used to establish some investigations related to the design of adaptive controller for a robot.

Dynamics of five-bar linkage: Consider the manipulator shown in Fig. 1. It is clear from the figure that even though there are four links being moved, there are in fact only two degrees-of-freedom, identified as $\varphi_{1}$ and $\varphi_{2}$. As the mechanism is a closed kinematics chain, Jacobian matrices are employed to work out the dynamic equations of the mechanism.

The coordinates of the centers of mass of four links as a function of the generalized coordinates are calculated as follows ${ }^{[3]}$ :

$$
\begin{aligned}
& {\left[\begin{array}{l}
x_{c 1} \\
y_{c 1}
\end{array}\right]=\left[\begin{array}{l}
l_{c 1} \cos \varphi_{1} \\
l_{c 1} \sin \varphi_{1}
\end{array}\right]} \\
& {\left[\begin{array}{l}
x_{c 2} \\
y_{c 2}
\end{array}\right]=\left[\begin{array}{l}
l_{c 2} \cos \varphi_{2} \\
l_{c 2} \sin \varphi_{2}
\end{array}\right]} \\
& {\left[\begin{array}{l}
x_{c 3} \\
y_{c 3}
\end{array}\right]=\left[\begin{array}{l}
l_{2} \cos \varphi_{2} \\
l_{2} \sin \varphi_{2}
\end{array}\right]+\left[\begin{array}{l}
l_{c 3} \cos \varphi_{1} \\
l_{c 3} \sin \varphi_{1}
\end{array}\right]} \\
& {\left[\begin{array}{l}
x_{c 4} \\
y_{c 4}
\end{array}\right]=\left[\begin{array}{l}
l_{1} \cos \varphi_{1} \\
l_{1} \sin \varphi_{1}
\end{array}\right]-\left[\begin{array}{l}
l_{c 4} \cos \varphi_{2} \\
l_{c 4} \sin \varphi_{2}
\end{array}\right]}
\end{aligned}
$$

By differentiating equations (1), (2), (3), (4), the velocities of the various centers of mass are determined w.r.t. \& and \&

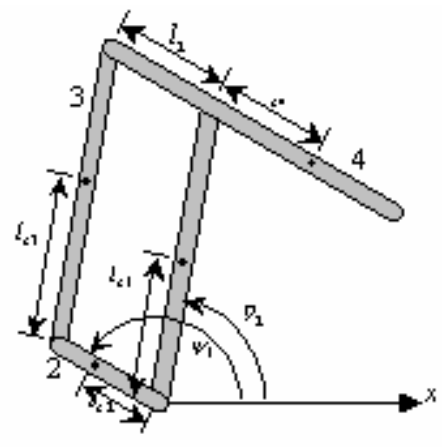

Fig. 1: Five-bar linkage mechanism

$$
V_{c i}=J_{i} \& i=1, \mathrm{~L}, 4
$$

where

$$
\begin{aligned}
& J_{1}=\left[\begin{array}{cc}
-l_{c 1} \sin \varphi_{1} & 0 \\
l_{c 1} \cos \varphi_{1} & 0
\end{array}\right] \\
& J_{2}=\left[\begin{array}{ll}
0 & -l_{c 2} \sin \varphi_{2} \\
0 & l_{c 2} \cos \varphi_{2}
\end{array}\right] \\
& J_{3}=\left[\begin{array}{ll}
-l_{c 3} \sin \varphi_{1} & -l_{2} \sin \varphi_{2} \\
l_{c 3} \cos \varphi_{1} & l_{2} \cos \varphi_{2}
\end{array}\right] \\
& J_{4}=\left[\begin{array}{cc}
-l_{1} \sin \varphi_{1} & l_{c 4} \sin \varphi_{2} \\
l_{1} \cos \varphi_{1} & -l_{c 4} \cos \varphi_{2}
\end{array}\right]
\end{aligned}
$$

The inertia matrix is given by

$$
M(\Phi)=\sum_{i=1}^{4} m_{i} J_{i}^{T} J_{i}+\left[\begin{array}{cc}
I_{1}+I_{3} & 0 \\
0 & I_{2}+I_{4}
\end{array}\right]
$$

Substituting equation (5) into (6), we obtain

$$
\begin{aligned}
& m_{11}(\Phi)=m_{1} l_{c 1}{ }^{2}+m_{3} l_{c 3}{ }^{2}+m_{4} l_{1}^{2}+I_{1}+I_{3} \\
& m_{12}(\Phi)=m_{21}(\Phi)=\left(m_{3} l_{2} l_{c 3}-m_{4} l_{1} l_{c 4}\right) \cos \left(\varphi_{2}-\varphi_{1}\right) \\
& m_{22}(\Phi)=m_{2} l_{c 2}{ }^{2}+m_{3} l_{2}{ }^{2}+m_{4} l_{c 4}{ }^{2}+I_{2}+I_{4}
\end{aligned}
$$

If the following simplification is assumed $m_{3} l_{2} l_{c 3}=m_{4} l_{1} l_{c 4}$ 
then the inertia matrix is diagonal and constant and consequently the dynamical equations will contain neither coriolis nor centripetal terms.

Using potential energy formulations, we have

$$
\begin{aligned}
E_{P}= & g \sin \varphi_{1}\left(m_{1} l_{c 1}+m_{3} l_{c 3}+m_{4} l_{1}\right) \\
& +g \sin \varphi_{2}\left(m_{2} l_{c 2}+m_{3} l_{2}-m_{4} l_{c 4}\right)
\end{aligned}
$$

Hence

$$
G(\Phi)=\frac{\partial E_{P}}{\partial \Phi}=\left[\begin{array}{c}
g \cos \varphi_{1}\left(m_{1} l_{c 1}+m_{3} l_{c 3}+m_{4} l_{1}\right) \\
g \cos \varphi_{2}\left(m_{2} l_{c 2}+m_{3} l_{2}-m_{4} l_{c 4}\right)
\end{array}\right]
$$

Based on Euler-Lagrange equation, the dynamic system of five-bar linkage robot can be represented as follow:

$$
\left\{\begin{array}{l}
m_{11}+g_{1}=\tau_{1} \\
m_{22}+g_{2}=\tau_{2}
\end{array}\right.
$$

Space-state model of equation (10) is expressed by

$$
\left\{\begin{array}{l}
\&=x_{2} \\
\&=-\theta_{1} \cos x_{1}+\theta_{2} u_{1} \\
\&=x_{4} \\
\&=-\theta_{3} \cos x_{3}+\theta_{4} u_{2}
\end{array}\right.
$$

where

$$
\begin{aligned}
& x_{1}=\varphi_{1}, x_{3}=\varphi_{3} \\
& u_{1}=\tau_{1}, u_{2}=\tau_{2} \\
& \theta_{1}=\frac{g\left(m_{1} l_{c 1}+m_{3} l_{c 3}+m_{4} l_{1}\right)}{m_{1} l_{c 1}{ }^{2}+m_{3} l_{c 3}{ }^{2}+m_{4} l_{1}{ }^{2}+I_{1}+I_{3}} \\
& \theta_{2}=\frac{1}{m_{1} l_{c 1}{ }^{2}+m_{3} l_{c 3}{ }^{2}+m_{4} l_{1}{ }^{2}+I_{1}+I_{3}} \\
& \theta_{3}=\frac{g\left(m_{2} l_{c 2}+m_{3} l_{2}-m_{4} l_{c 4}\right)}{m_{2} l_{c 2}{ }^{2}+m_{3} l_{2}{ }^{2}+m_{4} l_{c 4}{ }^{2}+I_{2}+I_{4}} \\
& \theta_{4}=\frac{1}{m_{2} l_{c 2}{ }^{2}+m_{3} l_{2}{ }^{2}+m_{4} l_{c 4}{ }^{2}+I_{2}+I_{4}}
\end{aligned}
$$

which will be used to design the adaptive controller.

Design of adaptive controller: The dynamic system of the robot can be represented by two identical SISO models hence it is sufficient to design an adaptive controller.

Theorem: Control input and adaptive laws (13) will guarantee the stability of assumed SISO model (12).

$$
\begin{aligned}
& \left\{\begin{array}{l}
\&=x_{2} \\
\&=-\theta_{1} \cos x_{1}+\theta_{2} u
\end{array}\right. \\
& \left\{\begin{array}{l}
u=\frac{\hat{\theta}_{1} \cos x_{1}+e_{1}}{\hat{\theta}_{2}}+k e_{2} \\
\theta_{1}^{\&}=\gamma_{1} e_{2} \cos x_{1} \\
\&=\gamma_{2} \frac{e_{2}\left(e_{1}+\hat{\theta}_{1} \cos x_{1}\right)}{\hat{\theta}_{2}}
\end{array}\right.
\end{aligned}
$$

where $e_{1}=x_{1 d}-x_{1}, \quad e_{2}=x_{2 d}-x_{2}$ and $k, \gamma_{1}, \gamma_{2}$ are positive constants. $\theta_{1}, \theta_{2}$ are real parameters and $\hat{\theta}_{1}, \hat{\theta}_{2}$ are the estimated parameters.

Proof: Assuming a Lyapunov function

$V=\frac{1}{2}\left(e_{1}^{2}+e_{2}^{2}+\frac{1}{\gamma_{1}} \theta_{1}^{\hat{\theta}}+\frac{1}{\gamma_{2}} \theta_{2}^{\hat{\theta}}\right)$

where $\theta_{1}^{0}=\theta_{1}-\hat{\theta}_{1}$ and $\theta_{2}^{0}=\theta_{2}-\hat{\theta}_{2}$.

Differentiating (14) with respect to time, we have

$$
I^{\&}=e_{1} \&+e_{2} \&+\frac{1}{\gamma_{1}} \theta_{1}^{\&} \&_{1}^{\&}+\frac{1}{\gamma_{2}} \theta_{2} \&
$$

Substituting (12) and (13) into (15), we obtain

$$
\begin{aligned}
& I^{\&}=e_{1} e_{2}-e_{2}\left(-\theta_{1} \cos x_{1}+\theta_{2}\left(\frac{\hat{\theta}_{1} \cos x_{1}+e_{1}}{\hat{\theta}_{2}}+k e_{2}\right)\right) \\
& -\theta_{1} \varepsilon_{2} \cos x_{1}+\theta_{2}^{0} \frac{e_{2}\left(e_{1}+\hat{\theta}_{1} \cos x_{1}\right)}{\hat{\theta}_{2}}
\end{aligned}
$$

Simplifying and rewriting (16), it can be shown that

$$
\begin{aligned}
& I \&=e_{1} e_{2}\left(1-\frac{\theta_{2}}{\hat{\theta}_{2}}+\frac{\theta_{2}^{0}}{\hat{\theta}_{2}}\right) \\
& -e_{2} \cos x_{1}\left(\frac{-\theta_{1} \hat{\theta}_{2}+\theta_{2} \hat{\theta}_{1}-\theta_{2} \hat{\theta}_{1}+\theta_{1} \hat{\theta}_{2}}{\hat{\theta}_{2}}\right)-k \theta_{2} e_{2}^{2}
\end{aligned}
$$

which can be reduced to $I^{\&}=-k \theta_{2} e_{2}^{2} \leq 0$

As this expression holds true, the stability of the SISO model (12) with the given adaptive laws and control input (13) will be assured.

\section{RESULTS}

The CAD model of a real five-bar linkage robot was designed using Solidworks ${ }^{\circledR}$ which is used for manufacturing and translation into SimMechanics ${ }^{\circledR}$. Figure 2 and 3 depict SimMechanics and the manufactured models respectively.

The proposed realistic and practical SimMechanics model is further employed to simulate the designed controller which is implemented using Simulink. The complete model of the system with random input is illustrated in Fig. 4.

Simulation results are shown for a desired configuration of $\varphi_{1}=30^{\circ}$ and $\varphi_{2}=-10^{\circ}$ in Fig. 5 . Figure 6 demonstrates the target tracking capability of the proposed system for random inputs. From Fig. 6 it can be easily realized that tracking error is infinitesimal which assures high performance of the designed controller. 


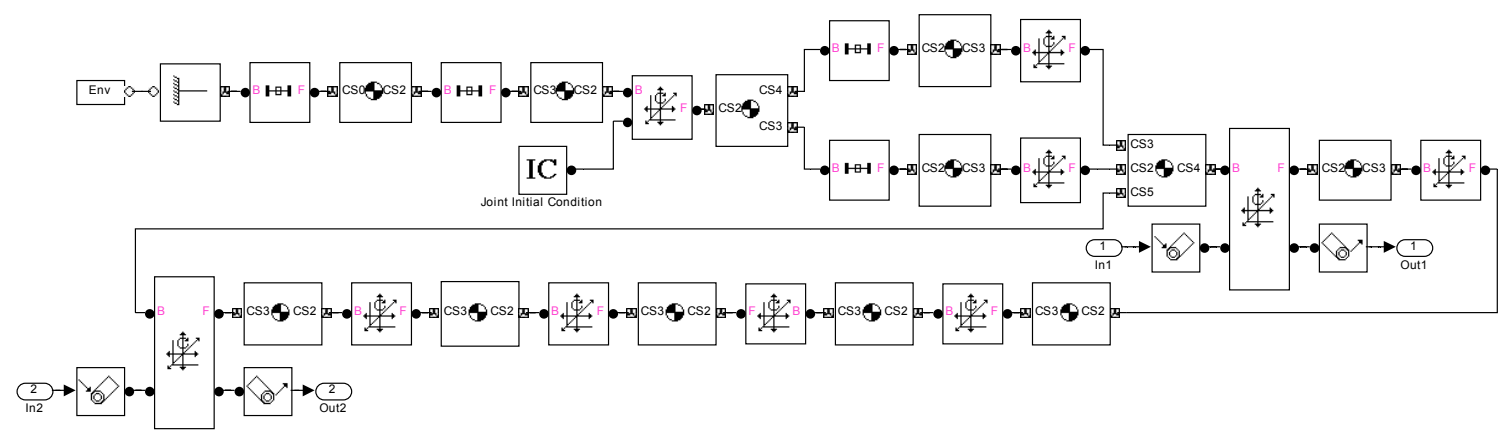

Fig. 2: Sim mechanics model of five-bar linkage

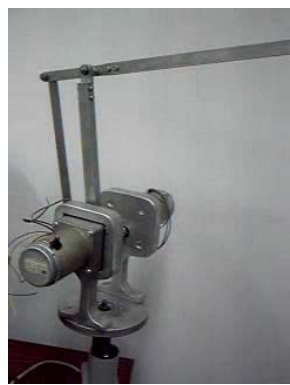

Fig. 3: Five-bar linkage robot

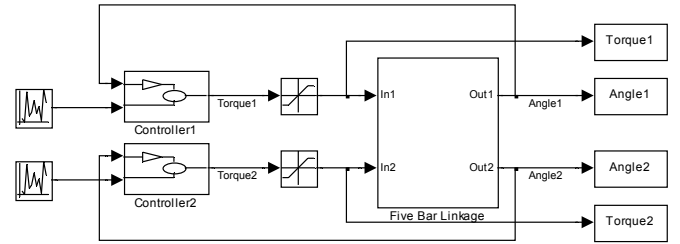

Fig. 4: Simulink model of the controllers and the robot
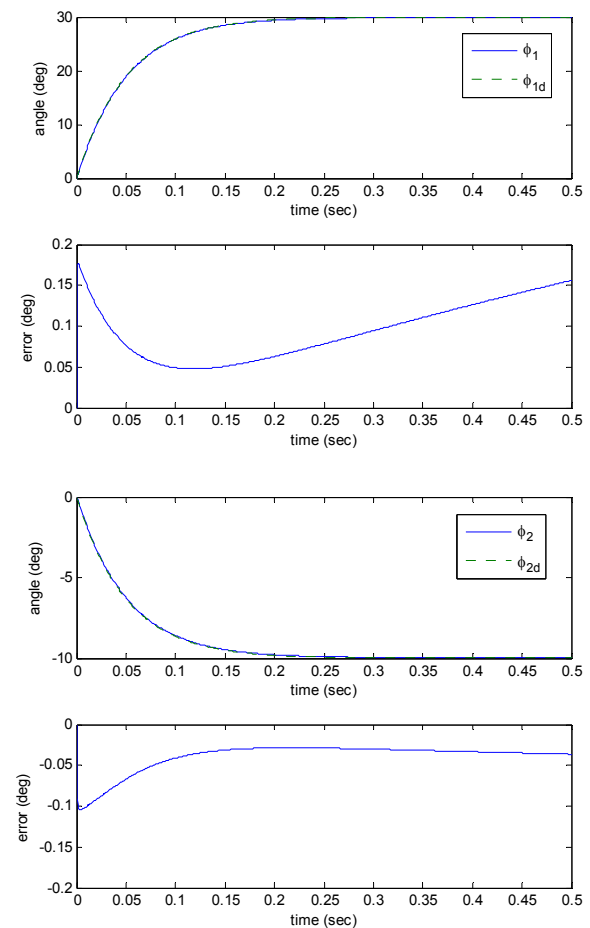

Fig. 5: Simulation results for $\varphi_{1}=30^{\circ}$ and $\varphi_{2}=-10^{\circ}$
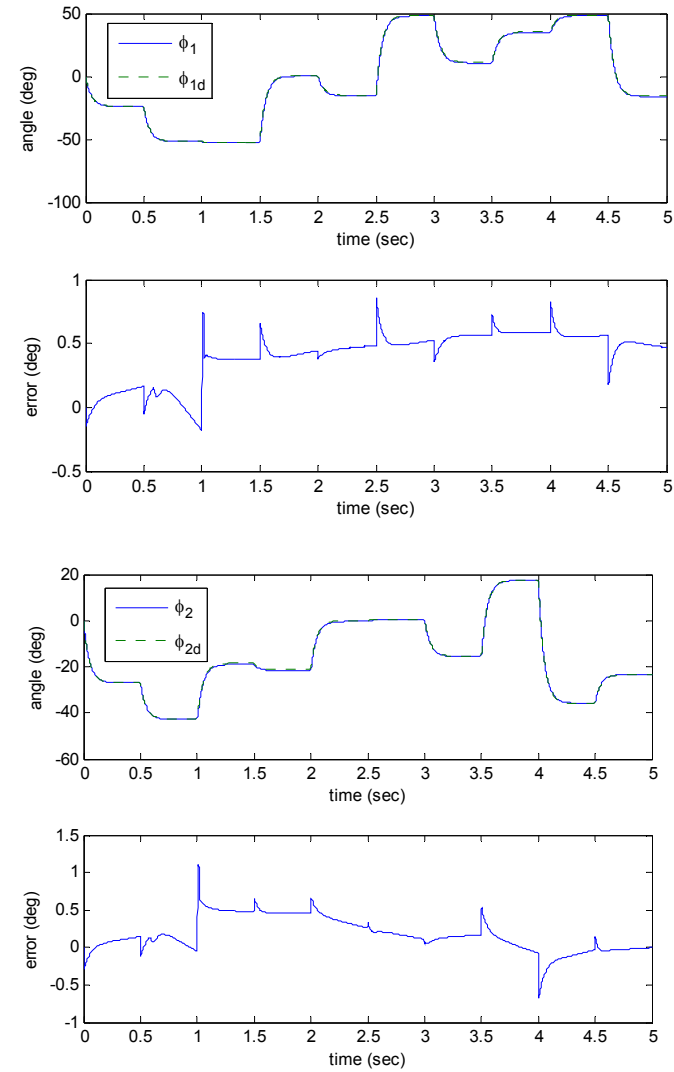

Fig. 6: Simulation results for random input

\section{CONCLUSION}

In this study the performance of an adaptive controller for five-bar linkage robot was investigated. The proposed controller is entirely independent on the physical specifications of the robot. Also, the stability of the proposed controller was verified. Simulation results have shown effectiveness of his controller. One of the most interesting things of this controller is that it is independent of the robot specifications in a wide range of variations as well as unstructured environment. IN the future we will implement the proposed method using HIL methods, i.e., XPC ${ }^{\circledR}$ target and RTWINT ${ }^{\circledR}$ toolboxes. Also it is extended to the third joint. 


\section{REFERENCES}

1. Spong, M.W. and M. Vidyasagar, 2006. Robot Dynamics and Control. New York, John Wiley and Sons, pp: $150-152$.

2. Hassanzadeh, I., M. Darabi and S. Hassanzadeh, 2006. Rapid prototyping of a manipulator mechanism using hardware in the loop (HIL) simulators and comparing the results.WSEAS Intl. Conf. ACMOS'06, Prague, Czech Republic, Mar. 12-14, 2006.

3. Hassanzadeh, I., S. Khanmohammadi and J. Jiang, Gh. Alizadeh, 2002. Implementation of a functional link Net_ANFIS controller for a robot manipulator. The $\overline{3}$ rd Intl. Workshop on Robot Motion and Control RoMoCo'02., pp: 404

4. Khanmohammadi, S., I. Hassanzadeh and M.B.B. Sharifian, 2000. Modified adaptive discrete control system containing neural estimator and neural controller. Elsevier, Artificial Intelligence in Engineering, 14: 1.
5. Slotine, J.J.E. and W. Li, 1988. Adaptive manipulator control: A case study. IEEE Trans. Automatic Control, 11: 1988.

6. Behera, L., S. Chaudhury and M. Gopal, 1998. Application of self-organizing neural networks in robot tracking control. IEEE Proc-Control Theory, 145: 135.

7. Kishan, K.K. and M. Jamshidi, 1997. Neural network based identification of robot dynamics used for Neuro_Fuzzy controller. Proc. IEEE Intl. Conf. Robotics and Automation Albuqureque, New Mexico, pp: 1118-1123.

8. QunhuaTan Weili, A hybrid neuro_fuzzy system for robot control. IEEE, pp: 2976-2921.

9. Pao, Y.H., 1990. Adaptive Pattern Recognition and Networks. Addison Wesley Publishing Company.

10. Kovacic, Z., V. Petik, T. Reichenbach and S. Bogdan, Robust self-learning fuzzy logic servo control with neural network-based load compensator. 